\title{
Relationship between serum concentrations of persistent organic pollutants and the prevalence of metabolic syndrome among non-diabetic adults: results from the National Health and Nutrition Examination Survey 1999-2002
}

\author{
D.-H. Lee • I.-K. Lee • M. Porta • M. Steffes • \\ D. R. Jacobs Jr
}

Received: 21 January 2007 / Accepted: 6 June 2007 /Published online: 12 July 2007

(C) Springer-Verlag 2007

\begin{abstract}
Aims/hypothesis We recently reported associations of some persistent organic pollutants (POPs) with both prevalence of type 2 diabetes and insulin resistance in a US population with background exposure to POPs. Restricted to nondiabetic participants, we now investigate the relationship
\end{abstract}

D.-H. Lee $(\bowtie)$

Department of Preventive Medicine and Health Promotion

Research Center, School of Medicine,

Kyungpook National University,

101 Dongin-dong, Jung-gu,

Daegu, 700-422 South Korea

e-mail: lee_dh@knu.ac.kr

I.-K. Lee

Department of Endocrinology, School of Medicine,

Kyungpook National University,

Daegu, South Korea

\section{Porta}

Institut Municipal d'Investigacio Medica,

Universitat Autonoma de Barcelona,

Barcelona, Spain

\section{Steffes}

Department of Laboratory Medicine and Pathology,

University of Minnesota,

Minneapolis, MN, USA

D. R. Jacobs Jr

Division of Epidemiology, School of Public Health,

University of Minnesota,

Minneapolis, MN, USA

D. R. Jacobs Jr

Department of Nutrition, University of Oslo,

Oslo, Norway between POPs and the metabolic syndrome, a prediabetic state.

Materials and methods Cross-sectional associations were investigated in 721 non-diabetic participants aged $\geq 20$ years. Nineteen POPs in five subclasses were selected because they were detectable in $\geq 60 \%$ of participants.

Results Among five POPs subclasses, organochlorine (OC) pesticides were most strongly and consistently associated with metabolic syndrome: adjusted odds ratios (ORs) of $1.0,1.5,2.3$ and 5.3 across OC pesticide quartiles ( $p$ for trend $<0.01)$. Dioxin-like polychlorinated biphenyls (PCBs) were also positively associated with adjusted ORs of 1.0, $1.1,2.2$ and 2.1 ( $p$ for trend=0.01). However, non-dioxinlike PCBs showed an inverted U-shaped association with adjusted ORs of 1.0, 1.3, 1.8 and 1.0 ( $p$ for quadratic term $<0.01)$. Associations of specific POPs varied across five components of the metabolic syndrome. OC pesticides were positively and significantly associated with four of the five components, especially elevated triacylglycerol and high fasting glucose, but not high blood pressure. PCBs were significantly associated with waist circumference, triacylglycerol and impaired fasting glucose. Polychlorinated dibenzo$p$-dioxins and polychlorinated dibenzofurans showed small but significant associations only with high blood pressure.

Conclusions/interpretation This study suggests that the prevalence of a cluster of cardiovascular risk factors relates to background exposure to a mixture of POPs, several of which are also related to the prevalence of diabetes. POPs associated differentially with different components of the metabolic syndrome.

Keywords Diabetes $\cdot$ Insulin resistance $\cdot$ Metabolic syndrome $\cdot$ Organochlorine pesticides $\cdot$ Persistent organic pollutants $\cdot$ Polychlorinated biphenyls 


\begin{tabular}{|c|c|}
\hline \multicolumn{2}{|c|}{ Abbreviations } \\
\hline HOMA-IR & $\begin{array}{l}\text { homeostasis model assessment of } \\
\text { insulin resistance }\end{array}$ \\
\hline LOD & limit of detection \\
\hline NHANES & National Health and Nutrition \\
\hline $\mathrm{OC}$ & $\begin{array}{l}\text { Examination Surveys } \\
\text { organochlorine }\end{array}$ \\
\hline OR & odds ratio \\
\hline PCB & polychlorinated biphenyl \\
\hline PCDD & polychlorinated dibenzo- $p$-dioxin \\
\hline PCDF & polychlorinated dibenzofuran \\
\hline POP & persistent organic pollutant \\
\hline
\end{tabular}

\section{Introduction}

The metabolic syndrome is characterised by the cooccurrence of obesity (especially central obesity), dyslipidaemia (especially high concentrations of triacylglycerol and low concentrations of HDL-cholesterol), hyperglycaemia and hypertension [1]. Although there is no consensus yet for specific thresholds for establishing the diagnosis of each of these traits as components of the syndrome, the individual traits of the syndrome cluster to a notably greater degree than expected by chance alone, a fact that lends substantial support to the existence of a discrete metabolically connected disorder $[1,2]$. People with metabolic syndrome are at increased risk of developing type 2 diabetes and cardiovascular diseases [3, 4].

We recently reported dose-response relationships between serum concentrations of persistent organic pollutants (POPs), notably organochlorine (OC) pesticides and polychlorinated biphenyl (PCB) congeners, and type 2 diabetes in a random sample of the US population with background environmental exposure to POPs [5]. In addition, when we assessed five subclasses of POPs among people without diabetes, we found that concentrations of $\mathrm{OC}$ pesticides and $\mathrm{PCBs}$ were positively related to the homeostasis model assessment of insulin resistance (HOMA-IR), suggestive of an increased risk of diabetes [6]. Furthermore, obesity was not associated with type 2 diabetes or insulin resistance among participants with very low levels of POPs, and the association between obesity and type 2 diabetes or insulin resistance became stronger as serum concentrations of POPs increased $[5,6]$. These findings raise the possibility that, in addition to obesity itself, some POPs stored in adipose tissues may be a key to the pathogenesis of insulin resistance and type 2 diabetes $[7,8]$.

Even though obesity, especially visceral obesity, has been regarded as a major factor affecting many components of the metabolic syndrome, our previous observations on POPs led us to hypothesise that the occurrence of metabolic syndrome may also be influenced by the background exposure to various POPs. Most human populations are exposed to mixtures of POPs, which include several hundred chemicals that bioaccumulate in fatty tissues of living organisms [6, 7]. Although specific types of POPs may have specific health effects, simultaneous exposure to various POPs through food consumption may lead to a cluster of health effects, such as metabolic syndrome.

In addition to our studies [5, 6], other studies in the USA and elsewhere have found the incidence or the prevalence of diabetes to be increased with increasing concentrations of some POPs [9-13] but, to the best of our knowledge, no studies have analysed the relationship with the metabolic syndrome. Thus, we investigated the relationship of serum concentrations of POPs with metabolic syndrome among non-diabetic participants using the same data-set as in our previous study of POPs and insulin resistance [6].

\section{Materials and methods}

Study population The 1999-2002 National Health and Nutrition Examination Surveys (NHANES) conducted by the Centers for Disease Control and Prevention were designed to be nationally representative of the non-institutionalised US civilian population on the basis of a complex, multistage probability sample. Details of the NHANES protocol and all testing procedures are available elsewhere $[14,15]$. Serum concentrations of various biologically important POPs or their metabolites were measured in subsamples of the NHANES 1999-2002 surveys [16].

Measurement The NHANES standardised home interview was followed by a detailed physical examination in a mobile evaluation clinic or the participant's home. Venous blood samples were collected and shipped weekly at $-20^{\circ} \mathrm{C}$. Waist circumference was measured at the high point of the iliac crest at minimal respiration to the nearest $0.1 \mathrm{~cm}$ at the end of normal expiration. Serum triacylglycerol concentration was measured enzymatically and HDL-cholesterol concentration, after the precipitation of other lipoproteins with a heparin-manganese chloride mixture, was measured on a Hitachi 717 Analyzer (Boehringer Mannheim Diagnostics, Indianapolis, IN, USA). Up to four blood pressure measurements were obtained from each participant. To establish high blood pressure status, we used the average of the last two measurements of blood pressure for participants who had three or four measurements, the last measurement for participants with only two measurements, and the only measurement for participants who had one measurement. Plasma glucose concentration was measured using an enzymatic reaction at the University of Missouri (Cobas 
Mira Chemistry System; Roche Diagnostic Systems, Montclair, NJ, USA).

POPs were measured by high-resolution gas chromatography/mass spectrometry using isotope dilution for quantification. All these analytes were measured in approximately $5 \mathrm{ml}$ serum using a modification of the method of Turner et al. [17]. Ability to detect low POP concentrations was greater in those participants who provided a larger sample. The POPs were reported on a lipid-adjusted basis using concentrations of serum total cholesterol and triacylglycerol. Plasma glucose was measured with a hexokinase enzymatic reference method (COBAS MIRA; Roche Diagnostics, Indianapolis, IN, USA), and serum insulin by means of an RIA (Pharmacia Diagnostics, Uppsala, Sweden).

Although 49 POPs were measured in both NHANES 1999-2000 and 2001-2002, to avoid bias in risk estimation among those below the limit of detection (LOD) we selected the 19 POPs for which at least $60 \%$ of study participants had concentrations more than the LOD: three polychlorinated dibenzo- $p$-dioxins (PCDDs), three polychlorinated dibenzofurans (PCDFs), four dioxin-like PCBs, five non-dioxin-like PCBs and four OC pesticides.

Final samples There were 852 study participants aged $\geq 20$ years with information on both fasting morning samples suitable for measurement of triacylglycerol and glucose and serum concentrations of the 19 selected POPs. After excluding 103 participants who had diabetes (fasting glucose concentration $\geq 7.0 \mathrm{mmol} / 1$ or history of physiciandiagnosed diabetes) and 28 participants with missing data on waist circumference, the final sample size was 721 .

Statistical methods We defined metabolic syndrome using the National Cholesterol Education Program definition. This definition was satisfied if a subject possessed three or more of the following five criteria: an abdominal waist circumference $>102 \mathrm{~cm}$ in men or $>88 \mathrm{~cm}$ in women; serum triacylglycerol $\geq 1.7 \mathrm{mmol} / \mathrm{l}$; serum HDL-cholesterol $<1.1 \mathrm{mmol} / 1$ in men or $<1.4 \mathrm{mmol} / 1$ in women; average blood pressure $\geq 130 / 85 \mathrm{mmHg}$, or currently taking antihypertensive medication; or fasting serum glucose $\geq 5.6 \mathrm{mmol} / \mathrm{l}$.

For each POP, participants with serum concentrations under the LOD were regarded as the reference group, and participants with detectable values categorised by cut-off points of 25th, 50th and 75th percentile values. To yield a cumulative measure of three PCDDs, we summed the ranks of the three POPs which belong to the PCDDs. The summary values were categorised by cut-off points of 25th, 50th and 75th percentile values. We assigned and cumulated POP subclasses similarly for the three PCDFs, the four dioxin-like PCBs, the five non-dioxin-like PCBs and the four $\mathrm{OC}$ pesticides.
Variables considered to be confounders in the multivariate analysis were age, sex, race/ethnicity, poverty income ratio, cigarette smoking (never, former or current), cotinine concentrations $(\mathrm{ng} / \mathrm{ml})$, alcohol consumption (g/day) and leisure time physical activity (vigorous, moderate or none). We further considered BMI as a possible confounder. We substituted median values of study participants for missing poverty income ratio, cotinine concentrations or alcohol consumption in 67 participants; exclusion of these individuals did not materially alter any estimates.

All statistical analyses were performed with SAS 9.1 (SAS Institute, Cary, NC, USA) and SUDAAN 9.0 (Research Triangle Institute, Research Triangle Park, NC, USA). Estimates of main results were calculated accounting for stratification and clustering [18], adjusting for age, race and ethnicity, and poverty income ratio instead of using sample weights; this adjustment has been regarded as a good compromise between efficiency and bias [18, 19]. As results were very similar with SAS 9.1 and SUDAAN 9.0, we present the results based on SAS 9.1.

\section{Results}

The sample of 721 participants included 175 persons with metabolic syndrome (prevalence 24.3\%). As previously reported [5, 6], age was the strongest and most important correlate of serum concentrations of all five subclasses of POPs (correlation coefficients $0.46-0.76, p<0.01$ ). After adjusting for age, there were positive pairwise correlations among serum concentrations of the five subclasses of POPs with correlation coefficients from 0.23 to 0.73 . Men had low serum concentrations of PCDDs, dioxin-like PCBs and OC pesticides. White participants had lower concentrations of OC pesticides, but higher concentrations of PCBs. Mexican Americans had the highest serum concentrations of OC pesticides. Those with lower income had higher concentrations of OC pesticides, but lower PCBs. Current smokers tended to have lower concentrations of most POPs, except non-dioxin-like PCBs. Alcohol consumption was positively associated with PCBs, while exercise was inversely associated with $\mathrm{OC}$ pesticides. OC pesticides were weakly and positively associated with BMI, while non-dioxin-like PCBs showed even an inverse association with BMI.

Table 1 shows associations between five subclasses of POPs and prevalence of metabolic syndrome. Among the five subclasses, dioxin-like PCBs, non-dioxin-like PCBs and $\mathrm{OC}$ pesticides were associated with metabolic syndrome. OC pesticides were most strongly and linearly associated with metabolic syndrome; adjusted odds ratios (ORs) were 1.0, 1.5, 2.3 and 5.3 ( $p$ for trend $<0.01$; model 2 
Table 1 Adjusted ORs and 95\% CIs of prevalence of metabolic syndrome by quartiles ${ }^{\mathrm{a}}$ of PCDDs, PCDFs, dioxin-like PCBs, non-dioxin-like PCBs and OC pesticides

\begin{tabular}{|c|c|c|c|c|c|}
\hline Analyte & $<25$ th & 25 th to $<50$ th & 50 th to $<75$ th & $\geq 75$ th & $p_{\text {trend }}$ \\
\hline \multicolumn{6}{|l|}{ PCDDs } \\
\hline Cases/no. & $29 / 180$ & $39 / 180$ & $50 / 181$ & $57 / 180$ & \\
\hline Model 1 & Referent & $1.2(0.7-2.1)$ & $1.4(0.8-2.3)$ & $1.2(0.7-2.3)$ & 0.46 \\
\hline Model 2 & Referent & $1.2(0.7-2.1)$ & $1.4(0.8-2.4)$ & $1.3(0.7-2.5)$ & 0.35 \\
\hline Model 3 & Referent & $1.1(0.6-2.0)$ & $1.1(0.6-2.1)$ & $1.0(0.5-2.0)$ & 0.95 \\
\hline \multicolumn{6}{|l|}{ PCDFs } \\
\hline Cases/no. & $30 / 180$ & $41 / 180$ & $48 / 181$ & $56 / 180$ & \\
\hline Model 1 & Referent & $1.3(0.7-2.2)$ & $1.3(0.8-2.3)$ & $1.4(0.8-2.4)$ & 0.29 \\
\hline Model 2 & Referent & $1.3(0.7-2.2)$ & $1.4(0.8-2.5)$ & $1.6(0.9-2.8)$ & 0.11 \\
\hline Model 3 & Referent & $1.5(0.8-2.7)$ & $1.4(0.8-2.6)$ & $1.6(0.8-3.1)$ & 0.21 \\
\hline \multicolumn{6}{|c|}{ Dioxin-like PCBs } \\
\hline Cases/no. & $27 / 180$ & $31 / 180$ & $56 / 181$ & $61 / 180$ & \\
\hline Model 1 & Referent & $1.0(0.6-1.8)$ & $1.7(1.0-3.1)$ & $1.5(0.8-3.0)$ & 0.09 \\
\hline Model 2 & Referent & $1.1(0.6-1.9)$ & $2.2(1.2-4.1)$ & $2.1(1.0-4.3)$ & 0.01 \\
\hline Model 3 & Referent & $1.1(0.6-2.1)$ & $2.2(1.1-4.3)$ & $2.3(1.1-5.1)$ & 0.01 \\
\hline \multicolumn{6}{|c|}{ Non-dioxin-like PCBs } \\
\hline Cases/no. & $29 / 180$ & $38 / 180$ & $61 / 181$ & $47 / 180$ & \\
\hline Model 1 & Referent & $1.1(0.7-2.0)$ & $1.4(0.8-2.4)$ & $0.7(0.4-1.4)$ & $0.02^{\mathrm{b}}$ \\
\hline Model 2 & Referent & $1.3(0.7-2.3)$ & $1.8(1.0-3.4)$ & $1.0(0.5-1.9)$ & $<0.01^{\mathrm{b}}$ \\
\hline Model 3 & Referent & $1.5(0.8-2.9)$ & $2.3(1.1-4.7)$ & $1.4(0.7-3.2)$ & $0.02^{\mathrm{b}}$ \\
\hline \multicolumn{6}{|c|}{ OC pesticides } \\
\hline Cases/no. & $20 / 180$ & $30 / 180$ & $46 / 181$ & $79 / 180$ & \\
\hline Model 1 & Referent & $1.6(0.8-2.9)$ & $2.6(1.4-5.0)$ & $5.9(2.8-12.2)$ & $<0.01$ \\
\hline Model 2 & Referent & $1.5(0.8-2.8)$ & $2.3(1.2-4.5)$ & $5.3(2.5-11.3)$ & $<0.01$ \\
\hline Model 3 & Referent & $1.5(0.7-3.1)$ & $2.0(0.9-4.3)$ & $4.8(2.0-11.3)$ & $<0.01$ \\
\hline
\end{tabular}

Model 1 Adjusted for age; model 2 additional adjustment for sex, race, poverty income ratio, cigarette smoking, serum cotinine, alcohol consumption and exercise; model 3 additional adjustment for BMI

${ }^{a}$ Detectable values of each POP were individually ranked and the rank orders of the individual POPs in each subclass were summed to arrive at the subclass value. All non-detectable values were ranked as 0 . The summary values were categorised by cut-off points of 25 th, 50 th and 75 th values of the sum of ranks

${ }^{\mathrm{b}} p$ values for quadratic term for non-dioxin-like PCBs

in Table 1). OC pesticides also showed ORs $>2$ for four of the five components of metabolic syndrome: waist circumference, elevated triacylglycerol, low HDL-cholesterol and high fasting glucose (Table 2). Particularly strong associations were observed with triacylglycerol and fasting glucose. PCBs tended to show non-linear dose-response relationships (Table 1). In the case of dioxin-like PCBs, the risk of metabolic syndrome reached the highest in the third quartile and then plateaued, leading to a $p$ for trend that was lower than the $p$ for a quadratic term; adjusted ORs were 1.0, 1.1, 2.2 and 2.1 ( $p$ for trend $=0.01$; model 2 in Table 1). However, non-dioxin-like PCBs showed a significant inverted U-shaped association with adjusted ORs of 1.0, $1.3,1.8$ and 1.0 ( $p$ for quadratic term $<0.01$; model 2 in Table 1). PCBs were linearly or quadratically associated with three of the five components: waist circumference, elevated triacylglycerol and high fasting glucose (Table 2). PCDDs and PCDFs were not associated with metabolic syndrome in Table 1, but these POPs were positively and significantly associated with high blood pressure (Table 2).
In addition, PCDDs showed a positive association with waist circumference. Further adjustment of BMI (model 3 in Table 1) did not materially change the results.

Detailed associations of the 19 individual POPs with metabolic syndrome were presented in Tables 3, 4, 5 and 6 . Although general trends of associations of individual POP appeared to be consistent with those of the subclasses shown in Tables 1 and 2, the associations did vary among specific POPs in the same POPs subclass.

Among three specific POPs belonging to PCDDs, only 1,2,3,4,6,7,8-heptachlorodibenzo- $p$-dioxin was significantly associated with waist circumference and high blood pressure (Table 3). Even though the subclass analyses of PCDFs failed to reach the statistical significance in the association with metabolic syndrome, 1,2,3,4,7,8-hexachlorodibenzofuran showed a significant positive association with metabolic syndrome as well as waist circumference and high blood pressure (Table 3).

Associations of specific dioxin-like PCB congeners with metabolic syndrome are summarised in Table 4. Congener 
Table 2 Adjusted ORs and 95\% CIs of prevalence of five components of the metabolic syndrome by quartiles ${ }^{\mathrm{a}}$ of PCDDs, PCDFs, dioxin-like PCBs, non-dioxin-like PCBs and OC pesticides

\begin{tabular}{|c|c|c|c|c|c|}
\hline & $<25$ th & 25 th to $<50$ th & 50 th to $<75$ th & $\geq 75$ th & $p_{\text {trend }}$ \\
\hline \multicolumn{6}{|c|}{ Waist circumference $>102 \mathrm{~cm}$ in men or $>88 \mathrm{~cm}$ in women } \\
\hline PCDDs & Referent & $1.2(0.8-1.9)$ & $1.4(0.9-2.3)$ & $1.8(1.0-3.0)$ & 0.03 \\
\hline PCDFs & Referent & $1.0(0.6-1.5)$ & $1.2(0.8-1.9)$ & $1.0(0.6-1.6)$ & 0.80 \\
\hline Dioxin-like PCBs & Referent & $1.0(0.7-1.6)$ & $1.7(1.0-2.9)$ & $1.6(0.9-2.9)$ & 0.05 \\
\hline Non-dioxin-like PCBs & Referent & $1.2(0.8-1.9)$ & $1.3(0.8-2.2)$ & $0.6(0.3-1.1)$ & $<0.01^{\mathrm{b}}$ \\
\hline OC pesticides & Referent & $1.2(0.8-1.9)$ & $1.7(1.0-2.9)$ & $2.4(1.3-4.6)$ & $<0.01$ \\
\hline \multicolumn{6}{|c|}{ Triacylglycerol $\geq 1.7 \mathrm{mmol} / 1$} \\
\hline PCDDs & Referent & $1.0(0.6-1.6)$ & $1.0(0.6-1.6)$ & $1.0(0.6-1.8)$ & 0.94 \\
\hline PCDFs & Referent & $0.9(0.6-1.4)$ & $1.0(0.6-1.6)$ & $0.7(0.4-1.2)$ & 0.26 \\
\hline Dioxin-like PCBs & Referent & $1.6(1.0-2.6)$ & $2.7(1.6-4.7)$ & $2.0(1.1-3.9)$ & 0.01 \\
\hline Non-dioxin-like PCBs & Referent & $1.6(1.0-2.7)$ & $2.0(1.1-3.5)$ & $1.7(0.9-3.2)$ & $0.05^{\mathrm{b}}$ \\
\hline OC pesticides & Referent & $1.4(0.8-2.5)$ & $3.1(1.8-5.5)$ & $7.1(3.6-14.1)$ & $<0.01$ \\
\hline \multicolumn{6}{|c|}{ HDL-cholesterol $<1.1 \mathrm{mmol} / 1$ in men or $<1.4 \mathrm{mmol} / 1$ in women } \\
\hline PCDDs & Referent & $0.8(0.5-1.2)$ & $0.7(0.4-1.1)$ & $0.8(0.5-1.5)$ & 0.38 \\
\hline PCDFs & Referent & $0.8(0.5-1.3)$ & $0.9(0.6-1.5)$ & $0.9(0.5-1.5)$ & 0.77 \\
\hline Dioxin-like PCBs & Referent & $1.1(0.7-1.8)$ & $1.3(0.7-2.1)$ & $1.1(0.6-2.1)$ & 0.65 \\
\hline Non-dioxin-like PCBs & Referent & $1.0(0.6-1.5)$ & $0.9(0.5-1.6)$ & $0.7(0.4-1.4)$ & $0.58^{\mathrm{b}}$ \\
\hline OC pesticides & Referent & $1.1(0.6-1.7)$ & $1.3(0.7-2.2)$ & $2.3(1.2-4.5)$ & 0.01 \\
\hline \multicolumn{6}{|c|}{ Blood pressure $\geq 130 / 85 \mathrm{mmHg}$} \\
\hline PCDDs & Referent & $1.1(0.7-1.9)$ & $1.4(0.8-2.3)$ & $1.7(1.0-3.1)$ & 0.04 \\
\hline PCDFs & Referent & $1.0(0.6-1.6)$ & $1.5(0.9-2.5)$ & $1.9(1.2-3.3)$ & $<0.01$ \\
\hline Dioxin-like PCBs & Referent & $1.1(0.7-1.9)$ & $0.9(0.5-1.6)$ & $1.4(0.8-2.7)$ & 0.42 \\
\hline Non-dioxin-like PCBs & Referent & $1.0(0.6-1.6)$ & $0.7(0.4-1.3)$ & $0.8(0.4-1.5)$ & $0.70^{\mathrm{b}}$ \\
\hline OC pesticides & Referent & $1.3(0.8-2.2)$ & $1.2(0.7-2.2)$ & $1.8(0.9-3.6)$ & 0.12 \\
\hline \multicolumn{6}{|c|}{ Fasting glucose $\geq 5.6 \mathrm{mmol} / \mathrm{l}$} \\
\hline PCDDs & Referent & $1.2(0.5-2.8)$ & $1.1(0.5-2.7)$ & $1.1(0.4-2.7)$ & 0.96 \\
\hline PCDFs & Referent & $1.2(0.5-2.9)$ & $1.5(0.6-3.6)$ & $1.6(0.6-3.7)$ & 0.28 \\
\hline Dioxin-like PCBs & Referent & $1.1(0.4-3.1)$ & $2.6(1.0-7.2)$ & $2.6(0.8-7.8)$ & 0.05 \\
\hline Non-dioxin-like PCBs & Referent & $1.5(0.5-4.1)$ & $2.5(0.9-6.8)$ & $1.3(0.4-4.0)$ & $0.03^{\mathrm{b}}$ \\
\hline OC pesticides & Referent & $3.2(0.9-11.9)$ & $3.6(0.9-13.8)$ & $5.6(1.4-23.0)$ & 0.02 \\
\hline
\end{tabular}

ORs adjusted for age, sex, race, poverty income ratio, cigarette smoking, serum cotinine, alcohol consumption and exercise

${ }^{\text {a }}$ Detectable values of each POP were individually ranked and the rank orders of the individual POPs in each subclass were summed to arrive at the subclass value. All non-detectable values were ranked as 0 . The summary values were categorised by cut-off points of 25 th, 50 th and 75 th values of the sum of ranks

${ }^{\mathrm{b}} p$ values for quadratic term for non-dioxin-like PCBs

126 was the most strongly and consistently associated with metabolic syndrome and its components. Congeners 74 and 118 had a substantial association with the syndrome but not so with the individual components. Congener 169 did not show any consistent pattern. The inverted $\mathrm{U}$-shaped associations of non-dioxin-like PCBs with metabolic syndrome, waist circumference, elevated triacylglycerol and high fasting glucose were consistently observed for all five specific congeners in the subclass (Table 5). This association was most strongly and consistently observed for elevated triacylglycerol.

Concerning OC pesticides, $\beta$-hexachlorocyclohexane showed the strongest association with metabolic syndrome as a whole (Table 6). However, specific OC pesticides appeared to have variable associations with different components of metabolic syndrome, the strongest being with increased fasting glucose.

\section{Discussion}

In this paper we continued our examination of the relationships between serum concentrations of POPs and the prevalence of diabetes [5] and insulin resistance [6] by studying associations between POP concentrations and the prevalence of metabolic syndrome among non-diabetic participants. Among five subclasses of POPs, dioxin-like PCBs, non-dioxin-like PCBs and OC pesticides were significantly associated with metabolic syndrome, although dose-response shapes varied depending on POPs subclass. In particular, OC pesticides were most strongly associated with metabolic syndrome with a linear dose-response relationship. Similarly to metabolic syndrome, OC pesticides were also most strongly associated with type 2 diabetes and insulin resistance in our previous studies $[5$, 
Table 3 Adjusted ORs and 95\% CIs of prevalence of metabolic syndrome by categories of specific POPs belonging to PCDDs and PCDFs

\begin{tabular}{|c|c|c|c|c|c|c|}
\hline & \multirow[t]{2}{*}{ Non-detectable } & \multicolumn{4}{|l|}{ Detectable } & \multirow[t]{2}{*}{$p_{\text {tren }}$} \\
\hline & & $<25$ th & 25 th to $<50$ th & 50 th to $<75$ th & $\geq 75$ th & \\
\hline \multicolumn{7}{|c|}{ Metabolic syndrome } \\
\hline D03 & Referent & $0.8(0.5-1.5)$ & $1.0(0.6-1.7)$ & $1.4(0.8-2.3)$ & $0.6(0.3-1.1)$ & 0.48 \\
\hline D05 & Referent & $1.5(0.7-3.0)$ & $2.0(1.0-4.0)$ & $1.5(0.7-3.1)$ & $2.0(0.9-4.1)$ & 0.13 \\
\hline D07 & Referent & $1.3(0.7-2.3)$ & $1.6(0.9-2.9)$ & $1.1(0.6-2.1)$ & $1.3(0.7-2.5)$ & 0.67 \\
\hline F03 & Referent & $1.7(1.0-3.0)$ & $1.4(0.8-2.4)$ & $1.4(0.8-2.6)$ & $1.0(0.5-1.8)$ & 0.56 \\
\hline F04 & Referent & $1.6(0.9-2.8)$ & $1.9(1.1-3.4)$ & $2.1(1.2-3.8)$ & $2.0(1.1-3.6)$ & $<0.01$ \\
\hline F08 & Referent & $1.2(0.7-2.1)$ & $0.9(0.5-1.6)$ & $1.1(0.6-2.0)$ & $1.0(0.6-1.7)$ & 0.87 \\
\hline \multicolumn{7}{|c|}{ Waist circumference $>102 \mathrm{~cm}$ in men or $>88 \mathrm{~cm}$ in women } \\
\hline D03 & Referent & $1.1(0.7-1.7)$ & $1.3(0.8-2.2)$ & $1.3(0.8-2.1)$ & $1.0(0.6-1.7)$ & 0.63 \\
\hline D05 & Referent & $1.6(0.9-2.7)$ & $1.4(0.8-2.4)$ & $1.9(1.1-3.4)$ & $2.6(1.4-4.9)$ & $<0.01$ \\
\hline D07 & Referent & $1.6(1.0-2.7)$ & $1.7(1.0-2.8)$ & $1.7(1.0-2.8)$ & $1.6(0.9-2.8)$ & 0.11 \\
\hline F03 & Referent & $2.0(1.2-3.2)$ & $1.3(0.8-2.1)$ & $1.0(0.6-1.7)$ & $0.7(0.4-1.3)$ & 0.56 \\
\hline F04 & Referent & $1.6(1.0-2.5)$ & $1.7(1.1-2.8)$ & $1.5(0.9-2.4)$ & $1.6(0.9-2.7)$ & 0.05 \\
\hline F08 & Referent & $1.1(0.7-1.8)$ & $0.9(0.6-1.5)$ & $0.9(0.6-1.5)$ & $0.9(0.5-1.4)$ & 0.41 \\
\hline \multicolumn{7}{|c|}{ Triacylglycerol $\geq 1.7 \mathrm{mmol} / 1$} \\
\hline D03 & Referent & $1.5(0.9-2.5)$ & $1.9(1.1-3.1)$ & $1.5(0.9-2.5)$ & $1.0(0.6-1.9)$ & 0.44 \\
\hline D05 & Referent & $2.1(1.2-3.8)$ & $1.5(0.9-2.8)$ & $1.0(0.5-1.8)$ & $1.2(0.6-2.4)$ & 0.47 \\
\hline D07 & Referent & $1.5(0.9-2.4)$ & $1.3(0.8-2.2)$ & $0.8(0.5-1.5)$ & $0.8(0.5-1.5)$ & 0.18 \\
\hline F03 & Referent & $1.9(1.2-3.1)$ & $1.2(0.8-2.0)$ & $1.3(0.8-2.2)$ & $0.7(0.4-1.2)$ & 0.70 \\
\hline F04 & Referent & $2.3(1.4-3.7)$ & $1.9(1.2-3.2)$ & $1.3(0.8-2.2)$ & $1.2(0.7-2.1)$ & 0.49 \\
\hline F08 & Referent & $1.6(1.0-2.5)$ & $0.6(0.4-1.0)$ & $0.9(0.5-1.4)$ & $0.5(0.3-0.8)$ & $<0.01$ \\
\hline \multicolumn{7}{|c|}{ HDL-cholesterol $<1.1 \mathrm{mmol} / 1$ in men or $<1.4 \mathrm{mmol} / 1$ in women } \\
\hline D03 & Referent & $0.9(0.5-1.4)$ & $0.8(0.5-1.3)$ & $1.0(0.6-1.7)$ & $0.5(0.3-1.0)$ & 0.13 \\
\hline D05 & Referent & $0.6(0.3-1.0)$ & $0.6(0.4-1.1)$ & $0.6(0.3-1.1)$ & $0.8(0.5-1.5)$ & 0.72 \\
\hline D07 & Referent & $0.9(0.6-1.5)$ & $0.9(0.5-1.5)$ & $0.5(0.3-0.9)$ & $0.9(0.5-1.6)$ & 0.18 \\
\hline F03 & Referent & $1.0(0.6-1.7)$ & $1.1(0.7-1.8)$ & $0.7(0.4-1.3)$ & $0.9(0.5-1.6)$ & 0.53 \\
\hline F04 & Referent & $0.9(0.5-1.4)$ & $0.8(0.5-1.4)$ & $1.3(0.8-2.1)$ & $1.2(0.7-2.0)$ & 0.48 \\
\hline F08 & Referent & $0.8(0.5-1.4)$ & $0.8(0.5-1.2)$ & $0.8(0.5-1.4)$ & $0.9(0.5-1.4)$ & 0.50 \\
\hline \multicolumn{7}{|c|}{ Blood pressure $\geq 130 / 85 \mathrm{mmHg}$} \\
\hline D03 & Referent & $0.7(0.4-1.3)$ & $0.8(0.5-1.5)$ & $1.3(0.8-2.1)$ & $1.2(0.7-2.2)$ & 0.33 \\
\hline D05 & Referent & $0.9(0.5-1.7)$ & $1.6(0.9-2.9)$ & $1.2(0.6-2.3)$ & $2.6(1.3-5.0)$ & $<0.01$ \\
\hline D07 & Referent & $0.7(0.4-1.2)$ & $1.0(0.6-1.8)$ & $1.2(0.7-2.1)$ & $1.1(0.6-2.0)$ & 0.29 \\
\hline F03 & Referent & $0.9(0.5-1.5)$ & $1.0(0.6-1.6)$ & $1.6(0.9-2.7)$ & $1.3(0.7-2.4)$ & 0.16 \\
\hline F04 & Referent & $0.8(0.5-1.4)$ & $1.6(1.0-2.7)$ & $2.0(1.2-3.4)$ & $2.3(1.3-4.0)$ & $<0.01$ \\
\hline F08 & Referent & $0.9(0.5-1.6)$ & $1.1(0.7-1.9)$ & $1.5(0.9-2.6)$ & $1.4(0.8-2.3)$ & 0.08 \\
\hline \multicolumn{7}{|c|}{ Fasting glucose $\geq 5.6 \mathrm{mmol} / 1$} \\
\hline D03 & Referent & $1.4(0.6-3.4)$ & $0.6(0.2-1.6)$ & $1.2(0.6-2.7)$ & $0.8(0.3-1.8)$ & 0.77 \\
\hline D05 & Referent & $1.1(0.4-3.0)$ & $1.1(0.4-3.0)$ & $1.0(0.4-2.8)$ & $1.1(0.4-3.1)$ & 0.96 \\
\hline D07 & Referent & $0.9(0.3-2.2)$ & $1.1(0.4-2.6)$ & $1.0(0.4-2.5)$ & $1.0(0.4-2.6)$ & 0.92 \\
\hline F03 & Referent & $1.1(0.4-3.1)$ & $0.9(0.4-2.1)$ & $2.3(1.1-4.7)$ & $1.1(0.5-2.6)$ & 0.31 \\
\hline F04 & Referent & $1.3(0.5-3.2)$ & $1.2(0.5-2.7)$ & $1.3(0.6-2.9)$ & $1.3(0.6-2.8)$ & 0.56 \\
\hline F08 & Referent & $1.2(0.5-2.9)$ & $1.7(0.8-3.8)$ & $1.2(0.5-2.8)$ & $1.1(0.5-2.5)$ & 0.89 \\
\hline
\end{tabular}

ORs adjusted for age, sex, race, poverty income ratio, cigarette smoking, serum cotinine, alcohol consumption and exercise

D03 1,2,3,6,7,8-hexachlorodibenzo-p-dioxin; D05 1,2,3,4,6,7,8-heptachlorodibenzo- $p$-dioxin; D07 1,2,3,4,6,7,8,9-octachlorodibenzo- $p$-dioxin; F03 2,3,4,7,8-pentachlorodibenzofuran; F04 1,2,3,4,7,8-hexachlorodibenzofuran; F08 1,2,3,4,6,7,8-heptachlorodibenzofuran

6]. However, dioxin-like PCBs showed a dose-response relationship with a plateau, while non-dioxin-like PCBs showed an inverted U-shaped relationship.

The inverted U-shaped associations observed with nondioxin-like PCBs deserve further discussion. Considering the consistency with all specific POPs belonging to nondioxin-like PCBs, this association may be real. This observation appears to be consistent with the association between POPs and diabetes, because this association was much steeper across lower background concentrations than across higher background concentrations [5, 7]. In agreement with this concept, others have reported that endocrine disrupters such as bisphenol A can show a non-monotonic dose-response or even an inverted U-shaped association 
Table 4 Adjusted ORs and 95\% CIs of prevalence of metabolic syndrome by categories of specific POPs belonging to dioxin-like PCBs

\begin{tabular}{|c|c|c|c|c|c|c|}
\hline & \multirow[t]{2}{*}{ Non-detectable } & \multicolumn{4}{|l|}{ Detectable } & \multirow[t]{2}{*}{$p_{\text {trend }}$} \\
\hline & & $<25$ th & 25 th to $<50$ th & 50 th to $<75$ th & $\geq 75$ th & \\
\hline \multicolumn{7}{|c|}{ Metabolic syndrome } \\
\hline PCB074 & Referent & $2.8(1.5-5.1)$ & $3.5(1.9-6.3)$ & $3.0(1.5-5.8)$ & $2.0(0.9-4.2)$ & $<0.01$ \\
\hline PCB118 & Referent & $2.9(1.6-5.1)$ & $1.7(0.9-3.2)$ & $2.4(1.3-4.4)$ & $2.5(1.3-5.1)$ & $<0.01$ \\
\hline PCB126 & Referent & $1.1(0.6-2.0)$ & $2.0(1.1-3.5)$ & $1.6(0.9-3.0)$ & $2.7(1.5-5.0)$ & $<0.01$ \\
\hline PCB169 & Referent & $0.7(0.4-1.3)$ & $1.0(0.5-1.7)$ & $1.0(0.5-1.9)$ & $0.4(0.2-0.8)$ & 0.10 \\
\hline \multicolumn{7}{|c|}{ Waist circumference $>102 \mathrm{~cm}$ in men or $>88 \mathrm{~cm}$ in women } \\
\hline PCB074 & Referent & $1.3(0.8-2.2)$ & $2.2(1.3-3.7)$ & $2.0(1.1-3.7)$ & $1.3(0.7-2.4)$ & 0.08 \\
\hline PCB118 & Referent & $1.5(0.9-2.4)$ & $1.9(1.1-3.1)$ & $1.6(1.0-2.8)$ & $2.2(1.2-4.1)$ & $<0.01$ \\
\hline PCB126 & Referent & $1.1(0.7-1.8)$ & $1.8(1.1-2.9)$ & $1.9(1.1-3.1)$ & $2.4(1.4-4.1)$ & $<0.01$ \\
\hline PCB169 & Referent & $1.1(0.7-1.8)$ & $0.7(0.4-1.1)$ & $0.9(0.5-1.5)$ & $0.3(0.1-0.5)$ & $<0.01$ \\
\hline \multicolumn{7}{|c|}{ Triacylglycerol $\geq 1.7 \mathrm{mmol} / 1$} \\
\hline РCB074 & Referent & $3.8(2.3-6.5)$ & $2.6(1.5-4.5)$ & $2.7(1.5-4.9)$ & $1.6(0.8-3.2)$ & 0.11 \\
\hline PCB118 & Referent & $4.9(2.9-8.2)$ & $2.3(1.3-4.1)$ & $2.8(1.6-5.0)$ & $3.0(1.6-5.8)$ & $<0.01$ \\
\hline PCB126 & Referent & $1.6(0.9-2.7)$ & $3.2(1.9-5.3)$ & $1.4(0.8-2.4)$ & $2.1(1.2-3.7)$ & 0.02 \\
\hline PCB169 & Referent & $1.5(0.9-2.4)$ & $1.1(0.6-1.8)$ & $1.2(0.7-2.1)$ & $0.8(0.4-1.5)$ & 0.50 \\
\hline \multicolumn{7}{|c|}{ HDL-cholesterol $<1.1 \mathrm{mmol} / 1$ in men or $<1.4 \mathrm{mmol} / 1$ in women } \\
\hline PCB074 & Referent & $1.3(0.8-2.2)$ & $1.9(1.1-3.2)$ & $1.1(0.6-2.1)$ & $1.1(0.6-2.2)$ & 0.52 \\
\hline PCB118 & Referent & $1.3(0.8-2.1)$ & $1.0(0.6-1.6)$ & $1.2(0.7-2.0)$ & $1.1(0.6-2.1)$ & 0.82 \\
\hline PCB126 & Referent & $1.2(0.7-2.0)$ & $1.4(0.9-2.3)$ & $1.2(0.7-2.1)$ & $1.7(1.0-3.0)$ & 0.09 \\
\hline PCB169 & Referent & $0.8(0.5-1.2)$ & $0.8(0.5-1.3)$ & $0.9(0.5-1.6)$ & $0.6(0.3-1.1)$ & 0.17 \\
\hline \multicolumn{7}{|c|}{ Blood pressure $\geq 130 / 85 \mathrm{mmHg}$} \\
\hline РCB074 & Referent & $0.8(0.5-1.4)$ & $1.2(0.7-2.1)$ & $0.9(0.5-1.7)$ & $1.2(0.6-2.4)$ & 0.57 \\
\hline PCB118 & Referent & $0.9(0.5-1.6)$ & $0.9(0.5-1.6)$ & $0.9(0.5-1.7)$ & $1.8(1.0-3.5)$ & 0.22 \\
\hline PCB126 & Referent & $0.6(0.3-1.1)$ & $1.2(0.7-2.0)$ & $1.0(0.6-1.7)$ & $2.1(1.2-3.7)$ & 0.02 \\
\hline PCB169 & Referent & $1.0(0.6-1.7)$ & $0.8(0.5-1.4)$ & $0.9(0.5-1.6)$ & $0.6(0.3-1.1)$ & 0.16 \\
\hline \multicolumn{7}{|c|}{ Fasting glucose $\geq 5.6 \mathrm{mmol} / 1$} \\
\hline PCB074 & Referent & $2.3(1.0-5.6)$ & $1.7(0.7-4.1)$ & $1.6(0.6-4.3)$ & $2.0(0.7-5.9)$ & 0.40 \\
\hline PCB118 & Referent & $1.6(0.6-3.8)$ & $2.2(0.9-5.3)$ & $1.6(0.6-4.2)$ & $2.7(1.0-7.5)$ & 0.09 \\
\hline PCB126 & Referent & $0.8(0.3-2.3)$ & $1.2(0.5-2.8)$ & $1.6(0.7-3.6)$ & $2.2(0.9-5.1)$ & 0.05 \\
\hline PCB169 & Referent & $1.2(0.4-3.4)$ & $1.5(0.6-3.9)$ & $1.3(0.5-3.3)$ & $0.9(0.3-2.6)$ & 0.79 \\
\hline
\end{tabular}

ORs adjusted for age, sex, race, poverty income ratio, cigarette smoking, serum cotinine, alcohol consumption and exercise

[20]; POPs are well-known endocrine disrupters. In addition, there was one experimental study in which increasing toxicity with increasing doses of some PCBs was even followed by a plateau or a decrease of toxicity in higher doses of them [21]. There should be further studies of the question whether exposure to low doses of PCBs can be more harmful than the exposure to high doses to PCBs.

However, there were some differences between the association of POPs with metabolic syndrome and the associations of POPs with either insulin resistance or type 2 diabetes. When specific POPs were individually analysed, chlordanes among the OC pesticides (oxychlordane and trans-nonachlor) were most strongly associated with both type 2 diabetes and insulin resistance $[5,6]$ while, in the case of metabolic syndrome, $\beta$-hexachlorocyclohexane was most strongly associated. However, when the five components of metabolic syndrome were separately examined, most OC pesticides appeared to be strongly associated with impaired fasting glucose, consistent with its relationship with insulin resistance or type 2 diabetes. The analysis of the specific and combined effects of POP mixtures and their components may be one of the greatest challenges in this area [7].

It has long been thought that insulin resistance may be the underlying pathophysiology in metabolic syndrome, as many of the components of the syndrome are associated with insulin resistance [22-24]. However, some recent studies [25-27] have reported that metabolic syndrome is not unequivocally related to insulin resistance. Even in this data-set, only $22.9 \%$ of subjects with metabolic syndrome had HOMA-IR $\geq 90$ th percentile; in our previous study [6], that high percentile cut-off point of HOMA-IR showed a stronger association with POPs than did lower cut-off points. Thus, some inconsistency of findings on metabolic syndrome vs those on insulin resistance or type 2 diabetes may be understandable. The findings also suggest that a mixture of POPs may be associated with a reinforcing set of metabolic abnormalities, probably not solely limited to diabetes. 
Table 5 Adjusted ORs and 95\% CIs of prevalence of metabolic syndrome by categories of specific POPs belonging to non-dioxin-like PCBs

\begin{tabular}{|c|c|c|c|c|c|c|}
\hline & \multirow[t]{2}{*}{ Non-detectable } & \multicolumn{4}{|l|}{ Detectable } & \multirow[t]{2}{*}{$p_{\text {quadratic }}{ }^{\mathrm{a}}$} \\
\hline & & $<25$ th & 25 th to $<50$ th & 50 th to $<75$ th & $\geq 75$ th & \\
\hline \multicolumn{7}{|c|}{ Metabolic syndrome } \\
\hline PCB138 & Referent & $1.3(0.7-2.4)$ & $1.8(1.0-3.1)$ & $1.4(0.8-2.7)$ & $1.1(0.6-2.1)$ & 0.03 \\
\hline PCB153 & Referent & $1.6(0.8-3.0)$ & $1.9(1.0-3.6)$ & $2.3(1.1-4.4)$ & $1.0(0.5-2.2)$ & $<0.01$ \\
\hline PCB170 & Referent & $1.7(0.9-3.0)$ & $2.3(1.2-4.2)$ & $1.4(0.7-2.7)$ & $0.8(0.4-1.7)$ & $<0.01$ \\
\hline PCB180 & Referent & $1.4(0.8-2.6)$ & $2.3(1.2-4.3)$ & $1.3(0.7-2.7)$ & $1.0(0.4-2.1)$ & $<0.01$ \\
\hline PCB187 & Referent & $2.6(1.5-4.6)$ & $1.9(1.0-3.6)$ & $1.3(0.7-2.6)$ & $1.3(0.7-2.7)$ & $<0.01$ \\
\hline \multicolumn{7}{|c|}{ Waist circumference $>102 \mathrm{~cm}$ in men or $>88 \mathrm{~cm}$ in women } \\
\hline PCB138 & Referent & $1.2(0.7-1.9)$ & $1.3(0.8-2.2)$ & $1.2(0.7-2.0)$ & $1.0(0.6-1.8)$ & 0.19 \\
\hline PCB153 & Referent & $1.5(0.9-2.5)$ & $1.3(0.8-2.3)$ & $1.5(0.8-2.6)$ & $0.8(0.4-1.4)$ & $<0.01$ \\
\hline PCB170 & Referent & $1.3(0.8-2.2)$ & $1.6(0.9-2.8)$ & $1.0(0.5-1.8)$ & $0.4(0.2-0.8)$ & $<0.01$ \\
\hline PCB180 & Referent & $1.3(0.8-2.1)$ & $0.9(0.5-1.6)$ & $0.9(0.5-1.7)$ & $0.4(0.2-0.7)$ & $<0.01$ \\
\hline PCB187 & Referent & $1.6(1.0-2.7)$ & $1.4(0.8-2.4)$ & $0.8(0.5-1.5)$ & $1.0(0.5-1.8)$ & 0.08 \\
\hline \multicolumn{7}{|c|}{ Triacylglycerol $\geq 1.7 \mathrm{mmol} / 1$} \\
\hline PCB138 & Referent & $2.0(1.2-3.4)$ & $2.1(1.2-3.6)$ & $2.4(1.4-4.2)$ & $1.5(0.8-2.8)$ & $<0.01$ \\
\hline PCB153 & Referent & $2.4(1.4-4.1)$ & $2.1(1.2-3.8)$ & $2.6(1.4-4.9)$ & $1.6(0.8-3.2)$ & $<0.01$ \\
\hline PCB170 & Referent & $2.6(1.6-4.4)$ & $1.9(1.1-3.3)$ & $1.6(0.9-3.1)$ & $1.5(0.8-3.0)$ & $<0.01$ \\
\hline PCB180 & Referent & $1.9(1.1-3.1)$ & $2.4(1.4-4.2)$ & $1.3(0.7-2.4)$ & $1.4(0.7-2.8)$ & $<0.01$ \\
\hline PCB187 & Referent & $5.0(2.9-8.6)$ & $2.1(1.1-3.7)$ & $2.2(1.2-4.2)$ & $1.7(0.9-3.3)$ & $<0.01$ \\
\hline \multicolumn{7}{|c|}{ HDL-cholesterol $<1.1 \mathrm{mmol} / 1$ in men or $<1.4 \mathrm{mmol} / 1$ in women } \\
\hline PCB138 & Referent & $0.8(0.5-1.4)$ & $0.8(0.5-1.4)$ & $1.0(0.6-1.7)$ & $0.7(0.4-1.3)$ & 0.98 \\
\hline PCB153 & Referent & $1.0(0.6-1.6)$ & $0.8(0.4-1.3)$ & $1.1(0.6-2.0)$ & $0.7(0.4-1.4)$ & 0.75 \\
\hline PCB170 & Referent & $1.0(0.6-1.7)$ & $1.0(0.6-1.7)$ & $1.1(0.6-2.0)$ & $0.6(0.3-1.3)$ & 0.22 \\
\hline PCB180 & Referent & $1.1(0.7-1.9)$ & $1.0(0.6-1.8)$ & $1.0(0.6-1.9)$ & $0.8(0.4-1.6)$ & 0.35 \\
\hline PCB187 & Referent & $1.0(0.6-1.7)$ & $0.8(0.5-1.5)$ & $0.8(0.4-1.5)$ & $0.9(0.5-1.6)$ & 0.80 \\
\hline \multicolumn{7}{|c|}{ Blood pressure $\geq 130 / 85 \mathrm{mmHg}$} \\
\hline PCB138 & Referent & $1.2(0.7-2.1)$ & $0.7(0.4-1.2)$ & $0.6(0.4-1.1)$ & $1.0(0.5-1.9)$ & 0.16 \\
\hline PCB153 & Referent & $0.9(0.5-1.6)$ & $0.8(0.5-1.5)$ & $0.6(0.3-1.1)$ & $0.8(0.4-1.5)$ & 0.52 \\
\hline PCB170 & Referent & $1.0(0.6-1.8)$ & $1.0(0.6-1.8)$ & $0.6(0.3-1.1)$ & $0.8(0.4-1.6)$ & 0.89 \\
\hline PCB180 & Referent & $0.8(0.5-1.4)$ & $1.2(0.7-2.1)$ & $0.7(0.4-1.3)$ & $0.8(0.4-1.7)$ & 0.83 \\
\hline PCB187 & Referent & $1.4(0.8-2.3)$ & $0.8(0.5-1.5)$ & $0.9(0.5-1.8)$ & $1.0(0.5-1.8)$ & 0.98 \\
\hline \multicolumn{7}{|c|}{ Fasting glucose $\geq 5.6 \mathrm{mmol} / \mathrm{l}$} \\
\hline PCB138 & Referent & $1.3(0.5-3.5)$ & $1.4(0.6-3.5)$ & $1.6(0.7-3.9)$ & $1.2(0.5-3.1)$ & 0.29 \\
\hline PCB153 & Referent & $1.3(0.4-4.1)$ & $2.5(0.9-7.0)$ & $2.6(0.9-7.5)$ & $1.7(0.5-5.4)$ & 0.05 \\
\hline PCB170 & Referent & $1.7(0.6-4.7)$ & $3.4(1.3-8.8)$ & $1.9(0.7-5.4)$ & $1.7(0.6-5.2)$ & 0.03 \\
\hline PCB180 & Referent & $1.6(0.5-4.9)$ & $2.5(0.9-7.1)$ & $2.5(0.8-7.7)$ & $1.9(0.6-6.2)$ & 0.08 \\
\hline PCB187 & Referent & $1.8(0.7-4.8)$ & $3.9(1.5-9.7)$ & $1.4(0.5-4.1)$ & $1.7(0.6-4.9)$ & 0.01 \\
\hline
\end{tabular}

ORs adjusted for age, sex, race, poverty income ratio, cigarette smoking, serum cotinine, alcohol consumption and exercise ${ }^{a} p$ values for quadratic term for non-dioxin-like PCBs

Importantly, subclasses of POPs were differently related to five components of metabolic syndrome. Especially, the POPs associations with blood pressure differed from those with metabolic syndrome traits more closely associated with diabetes. Differently from other components of the metabolic syndrome, high blood pressure was most strongly associated with PCDFs. On the other hand, OC pesticides were significantly associated with waist circumference, elevated triacylglycerol, low HDL-cholesterol and impaired fasting glucose, but showed only a non-significant positive trend with high blood pressure. Interestingly, factor analyses of traits of metabolic syndrome consistently show that blood pressure elevation clusters less closely than other traits in metabolic syndrome [28].

Although we examined specific POPs to know which were most strongly associated with metabolic syndrome itself or each component of the metabolic syndrome, only very large studies would have the ability to identify which POPs are aetiologically important and which are just correlated bystanders. Furthermore, since mechanistic studies in humans are constrained by ethical principles, knowledge of putative mechanisms for the observed relationships will require a variety of scientific approaches. Thus, our current findings should be regarded as tentative 
Table 6 Adjusted ORs and 95\% CIs of prevalence of metabolic syndrome by categories of specific POPs belonging to OC pesticides

\begin{tabular}{|c|c|c|c|c|c|c|}
\hline & \multirow[t]{2}{*}{ Non-detectable } & \multicolumn{4}{|l|}{ Detectable } & \multirow[t]{2}{*}{$p_{\text {trenc }}$} \\
\hline & & $<25$ th & 25 th to $<50$ th & 50 th to $<75$ th & $\geq 75$ th & \\
\hline \multicolumn{7}{|l|}{ Metabolic syndrome } \\
\hline Oxychlordane & Referent & $1.3(0.7-2.5)$ & $1.6(0.8-3.3)$ & $1.6(0.8-3.4)$ & $1.9(0.8-4.2)$ & 0.12 \\
\hline trans-Nonachlor & Referent & $1.3(0.6-2.8)$ & $1.3(0.6-2.9)$ & $1.5(0.7-3.4)$ & $1.8(0.8-4.3)$ & 0.17 \\
\hline$p, p^{\prime}-\mathrm{DDE}$ & & Referent & $1.0(0.5-1.7)$ & $1.7(0.9-2.9)$ & $1.0(0.5-1.9)$ & 0.67 \\
\hline Beta-HCH & Referent & $2.0(1.0-3.8)$ & $3.0(1.5-5.8)$ & $3.0(1.5-6.2)$ & $3.1(1.4-6.5)$ & $<0.01$ \\
\hline \multicolumn{7}{|c|}{ Waist circumference $>102 \mathrm{~cm}$ in men or $>88 \mathrm{~cm}$ in women } \\
\hline Oxychlordane & Referent & $1.4(0.9-2.4)$ & $1.5(0.9-2.5)$ & $1.4(0.8-2.6)$ & $1.2(0.6-2.4)$ & 0.57 \\
\hline trans-Nonachlor & Referent & $1.4(0.8-2.6)$ & $1.5(0.8-2.8)$ & $1.3(0.7-2.5)$ & $1.4(0.7-2.9)$ & 0.64 \\
\hline$p, p^{\prime}$-DDE & & Referent & $1.0(0.6-1.5)$ & $1.2(0.7-2.0)$ & $1.2(0.7-2.0)$ & 0.40 \\
\hline Beta-HCH & Referent & $1.0(0.6-1.7)$ & $1.1(0.7-1.9)$ & $1.6(0.9-2.9)$ & $1.6(0.9-3.0)$ & 0.07 \\
\hline \multicolumn{7}{|c|}{ Triacylglycerol $\geq 1.7 \mathrm{mmol} / 1$} \\
\hline Oxychlordane & Referent & $1.6(0.9-2.8)$ & $2.3(1.3-4.2)$ & $2.1(1.1-4.1)$ & $2.8(1.3-5.8)$ & $<0.01$ \\
\hline Trans-Nonachlor & Referent & $1.4(0.8-2.6)$ & $1.3(0.6-2.4)$ & $1.4(0.7-2.8)$ & $1.8(0.9-3.9)$ & 0.17 \\
\hline$p, p^{\prime}$-DDE & & Referent & $0.8(0.5-1.3)$ & $0.8(0.5-1.3)$ & $0.6(0.3-1.1)$ & 0.09 \\
\hline Beta-HCH & Referent & $2.7(1.6-4.6)$ & $2.6(1.5-4.6)$ & $2.3(1.2-4.2)$ & $2.1(1.1-4.0)$ & 0.08 \\
\hline \multicolumn{7}{|c|}{ HDL-cholesterol $<1.1 \mathrm{mmol} / 1 \mathrm{in}$ men or $<1.4 \mathrm{mmol} / 1$ in women } \\
\hline Oxychlordane & Referent & $1.0(0.6-1.6)$ & $1.2(0.7-2.1)$ & $0.9(0.5-1.7)$ & $1.3(0.6-2.6)$ & 0.63 \\
\hline trans-Nonachlor & Referent & $0.8(0.4-1.4)$ & $0.9(0.5-1.7)$ & $0.8(0.4-1.5)$ & $1.1(0.5-2.3)$ & 0.69 \\
\hline$p, p^{\prime}-\mathrm{DDE}$ & & Referent & $0.9(0.6-1.5)$ & $1.0(0.6-1.6)$ & $0.8(0.5-1.5)$ & 0.60 \\
\hline Beta-HCH & Referent & $1.1(0.6-1.8)$ & $1.1(0.7-2.0)$ & $1.1(0.6-2.1)$ & $1.4(0.7-2.5)$ & 0.39 \\
\hline \multicolumn{7}{|c|}{ Blood pressure $\geq 130 / 85 \mathrm{mmHg}$} \\
\hline Oxychlordane & Referent & $2.0(1.1-3.6)$ & $1.1(0.6-2.1)$ & $1.3(0.7-2.6)$ & $1.7(0.8-3.6)$ & 0.54 \\
\hline trans-Nonachlor & Referent & $1.2(0.6-2.4)$ & $1.2(0.6-2.4)$ & $1.7(0.8-3.7)$ & $1.4(0.6-3.2)$ & 0.18 \\
\hline$p, p^{\prime}-\mathrm{DDE}$ & & Referent & $1.0(0.6-1.6)$ & $1.3(0.8-2.2)$ & $1.3(0.7-2.4)$ & 0.23 \\
\hline Beta-HCH & Referent & $1.2(0.7-2.1)$ & $1.2(0.7-2.1)$ & $1.8(1.0-3.4)$ & $1.7(0.9-3.5)$ & 0.07 \\
\hline \multicolumn{7}{|c|}{ Fasting glucose $\geq 5.6 \mathrm{mmol} / \mathrm{l}$} \\
\hline Oxychlordane & Referent & $1.4(0.4-5.2)$ & $2.8(0.8-9.5)$ & $2.5(0.7-8.9)$ & $3.1(0.8-11.9)$ & 0.12 \\
\hline trans-Nonachlor & Referent & $4.0(0.5-32.8)$ & $5.0(0.6-41.0)$ & $5.3(0.6-44.7)$ & $7.0(0.8-59.6)$ & 0.09 \\
\hline$p, p^{\prime}-\mathrm{DDE}$ & & Referent & $4.4(1.2-15.7)$ & $3.7(1.0-13.3)$ & $6.6(1.8-24.3)$ & $<0.01$ \\
\hline Beta-HCH & Referent & $3.1(0.9-10.0)$ & $3.4(1.0-11.3)$ & $2.3(0.7-8.2)$ & $4.0(1.1-14.3)$ & 0.14 \\
\hline
\end{tabular}

ORs adjusted for age, sex, race, poverty income ratio, cigarette smoking, serum cotinine, alcohol consumption and exercise $p, p^{\prime}$-DDE, $p, p^{\prime}$-dichlorodiphenyltrichloroethane; beta-HCH, $\beta$-hexachlorocyclohexane

preliminary observations that add to existing knowledge, but require several types of additional confirmation such as mechanistic studies in animals, clinical studies and further epidemiological evidence from longitudinal designs with repeated measures $[7,8]$.

It may seem paradoxical that the strong associations between serum concentrations of POPs and metabolic syndrome, insulin resistance or type 2 diabetes are found when these clinical outcomes have recently become epidemic, while serum concentrations of some POPs have shown decreasing trends [8, 29]. In our previous studies [5, 6], we hypothesised a possible interaction with obesity to help explain these apparently conflicting trends: the toxicity of POPs may synergistically increase as people get obese. This idea was supported by recent molecular epidemiological evidence for diabetogenic effects of dioxin exposure; an inverse correlation between the ratio of GLUT4 to nuclear factor kappa B and serum dioxin concentrations was particularly significant among those with known risk factors of type 2 diabetes such as obesity and family history of diabetes [30]. In addition, other chemicals with similar properties to POPs, like the brominated flame retardants, perfluorinated compounds, which are still in widespread use today, may be as important as POPs which were measured in the NHANES [31]. The non-linear doseresponse with some PCBs may also play a role in the recent epidemic.

A further consideration is that the current generation may be experiencing epigenetic changes due to POPs in utero or even from altered ovum or sperm of their parents or grandparents; the fetal exposure to environmental pollutants such as POPs can cause epigenetic changes with transgenerational effects [32, 33]. Because POPs only began wide use after the Second World War [34], people who lived during that early time of POPs usage may have had little genetic effect due to in utero exposure to POPs 
even though their serum concentrations of POPs would be very high. However, in the current era, the cumulating exposure of POPs over several generations could be reflected in more epigenetic changes. Therefore we hypothesise that epigenetic changes have occurred and that the exposure to low background amounts of POPs in the current generation may be more harmful than the exposure to high amounts of POPs was in their parents. In fact, epigenetic alterations have been proposed as one important mechanism in the pathogenesis of type 2 diabetes or atherosclerosis [35]. Although this gene-environment interaction hypothesis goes beyond the data reported in this and our related papers based on the NHANES database, this possibility should be investigated in further studies.

Inuits are among the populations most highly exposed to POPs, mainly through the consumption of seal and beluga fat in the Arctic [36]. The prevalence of diabetes was extremely low in Inuit populations several decades ago, but diabetes, insulin resistance and metabolic syndrome have recently increased to the levels of other Westernised populations in some studies [37,38]. Even though both genetic susceptibility and rapid change of lifestyle have been named as causes, POPs stored in their adipose tissue may play an important role. Furthermore, epidemics of type 2 diabetes in many Asian countries could also be explained by POPs [39]. Although these chemicals were banned from manufacture and use in many developed countries during the 1970s, a number of them continue to be manufactured, stored, used and traded freely in developing Asian countries [40]. Perhaps it is not coincidental that India, China and Mexico are three of the largest remaining POP producers in the world [40].

This study has several limitations. First, the crosssectional study design in NHANES does not allow inferences regarding the causality between POPs and metabolic syndrome. One possibility is that disease-induced changes in body weight could cause bias if POP concentrations were differentially affected by such changes shortly before blood extraction. However, in the NHANES, there were no differences in changes in body weight between participants with and without metabolic syndrome. Thus, adjustment for changes in body weight did not change the results. Second, misclassification bias is possible for participants whose POPs would have been detectable with a higher sample volume. Such misclassification would be non-differential if sample volume is unrelated to metabolic syndrome. Third, inference should be made cautiously in light of the multiple comparisons intrinsic in this investigation, even though consistency of association of POPs with metabolic syndrome components reinforces the findings for metabolic syndrome.

In summary, along with our previous findings on the association of POPs with type 2 diabetes and insulin resistance, our current study suggests that the background exposure to some POPs may be closely related to metabolic syndrome, with different POPs related to different metabolic syndrome traits. Furthermore, the possibility of synergistic effects, where multiple POPs reinforce each other's toxicity in the general population, should be considered. These data lead to a hypothesis in which various POPs stored in adipose tissue may contribute to clustering of risk factors in metabolic syndrome.

Acknowledgements This study was partly supported by the Korea Science and Engineering Foundation through the NRL Program (Grant 2006-00562).

Duality of interest The authors declare that there is no duality of interest associated with this manuscript.

\section{References}

1. Wilson PWF, Kannel WB, Silbershatz H, D'Agostino RB (1999) Clustering of metabolic factors and coronary heart disease. Arch Intern Med 159:1104-1109

2. Pladevall M, Singal B, Williams LK et al (2006) A single factor underlies the metabolic syndrome: a confirmatory factor analysis. Diabetes Care 29:113-122

3. Hanson RL, Imperatore G, Bennett PH, Knowler WC (2002) Components of the 'metabolic syndrome' and incidence of type 2 diabetes. Diabetes 51:3120-3127

4. Lakka HM, Laaksonen DE, Lakka TA et al (2002) The metabolic syndrome and total and cardiovascular disease mortality in middle-aged men. JAMA 288:2709-2716

5. Lee DH, Lee IK, Song KE et al (2006) A strong dose-response relation between serum concentrations of persistent organic pollutants and diabetes: results from the National Health and Examination Survey. Diabetes Care 29:1638-1644

6. Lee DH, Lee IK, Jin SH, Steffes M, Jacobs DR Jr (2007) Association between serum concentrations of persistent organic pollutants and insulin resistance among nondiabetic adults: results from the National Health and Nutrition Examination Survey 1999-2002. Diabetes Care 30:622-628

7. Lee DH, Jacobs DR, Porta M (2006) Could low level background exposure to persistent organic pollutants contribute to the social burden of type 2 diabetes? [Editorial]. J Epidemiol Community Health 60:1006-1008

8. Porta M (2006) Persistent organic pollutants and the burden of diabetes. Lancet 368:558-559

9. Fierens S, Mairesse H, Heilier JF et al (2003) Dioxin/polychlorinated biphenyl body burden, diabetes and endometriosis: findings in a population-based study in Belgium. Biomarkers 8:529-534

10. Remillard RB, Bunce NJ (2002) Linking dioxins to diabetes: epidemiology and biologic plausibility. Environ Health Perspect 110:853-858

11. Steenland K, Calvert G, Ketchum N, Michalek J (2001) Dioxin and diabetes mellitus: an analysis of the combined NIOSH and Ranch Hand data. Occup Environ Med 58:641-648

12. Longnecker MP, Klebanoff MA, Brock JW, Zhou H (2001) Collaborative Perinatal Project (CPP). Polychlorinated biphenyl serum levels in pregnant subjects with diabetes. Diabetes Care 24:1099-1101

13. Cranmer M, Louie S, Kennedy RH, Kern PA, Fonseca VA (2000) Exposure to 2,3,7,8-tetrachlorodibenzo- $p$-dioxin (TCDD) is asso- 
ciated with hyperinsulinemia and insulin resistance. Toxicol Sci $56: 431-436$

14. NHANES 1999-2000 public data release file documentation [article online] (2005) Available from http://www.cdc.gov/nchs/ about/major/nhanes/nhanes99-00.htm, accessed 28 February 2006

15. NHANES 2000-2001 public data release file documentation [article online] (2005) Available from http://www.cdc.gov/nchs/ about/major/nhanes/nhanes01-02.htm, accessed 28 February 2006

16. Third national report on human exposure to environmental chemicals (2005) Available from http://www.cdc.gov/exposurereport/3rd/ pdf/thirdreport.pdf, accessed 28 February 2006

17. Turner W, DiPietro E, Lapeza C, Green V, Gill J, Patterson DG Jr (1997) A fast universal automated cleanup system for the isotopedilution HRMS analysis of PCDDs, PCDFs, coplanar PCBs, PCB congeners, and persistent pesticides from the same serum sample. Organohalog Compd 31:26-31

18. Korn EL, Graubard BI (1991) Epidemiologic studies utilizing surveys: accounting for the sampling design. Am J Public Health 81:1166-1173

19. Graubard BI, Korn EL (1999) Analyzing health surveys for cancer-related objectives. J Natl Cancer Inst 91:1005-1016

20. Welshons WV, Thayer KA, Judy BM, Taylor JA, Curran EM, vom Saal FS (2003) Large effects from small exposures. I. Mechanisms for endocrine-disrupting chemicals with estrogenic activity. Environ Health Perspect 111:994-1006

21. Hennig B, Meerarani P, Slim R (2002) Proinflammatory properties of coplanar PCBs: in vitro and in vivo evidence. Toxicol Appl Pharmacol 181:174-183

22. Grundy SM (1999) Hypertriglyceridemia, insulin resistance, and the metabolic syndrome. Am J Cardiol 83:25F-29F

23. Lillioja S, Mott DM, Spraul M et al (1993) Insulin resistance and insulin secretory dysfunction as precursors of non-insulin-dependent diabetes mellitus: prospective studies of Pima Indians. N Engl J Med 329:1988-1992

24. Warram JH, Martin BC, Krolewski AS, Soeldner JS, Kahn CR (1990) Slow glucose removal rate and hyperinsulinemia precede the development of type II diabetes in the offspring of diabetic parents. Ann Intern Med 113:909-915

25. Cheal KL, Abbasi F, Lamendola C, McLaughlin T, Reaven GM, Ford ES (2004) Relationship to insulin resistance of the adult treatment panel III diagnostic criteria for identification of the metabolic syndrome. Diabetes 53:1195-1200

26. Liao Y, Kwon S, Shaughnessy S et al (2004) Critical evaluation of adult treatment panel III criteria in identifying insulin resistance with dyslipidemia. Diabetes Care 27:978-983
27. McLaughlin T, Abbasi F, Cheal K, Chu J, Lamendola C, Reaven G (2003) Use of metabolic markers to identify overweight individuals who are insulin resistant. Ann Intern Med 139:802-809

28. Meigs JB (2000) Invited commentary: insulin resistance syndrome? Syndrome X? Multiple metabolic syndrome? A syndrome at all? Factor analysis reveals patterns in the fabric of correlated metabolic risk factors. Am J Epidemiol 152:908-911

29. Needham LL, Barr DB, Caudill SP et al (2005) Concentrations of environmental chemicals associated with neurodevelopmental effects in U.S. population. Neurotoxicology 26:531-545

30. Fujiyoshi PT, Michalek JE, Matsumura F (2006) Molecular epidemiologic evidence for diabetogenic effects of dioxin exposure in U.S. Air force veterans of the Vietnam war. Environ Health Perspect 114:1677-1683

31. Luross JM, Alaee M, Sergeant DB et al (2002) Spatial distribution of polybrominated diphenyl ethers and polybrominated biphenyls in lake trout from the Laurentian Great Lakes. Chemosphere 46:665-672

32. Anway MD, Cupp AS, Uzumcu M, Skinner MK (2005) Epigenetic transgenerational actions of endocrine disruptors and male fertility. Science 308:1466-1469

33. Titus-Ernstoff L, Troisi R, Hatch EE et al (2006) Menstrual and reproductive characteristics of women whose mothers were exposed in utero to diethylstilbestrol (DES). Int $\mathrm{J}$ Epidemiol $35: 862-868$

34. Thornton J (2000) Beyond risk: an ecological paradigm to prevent global chemical pollution. Int J Occup Environ Health 6:318330

35. Gallou-Kabani C, Junien C (2005) Nutritional epigenomics of metabolic syndrome: new perspective against the epidemic. Diabetes 54:1899-1906

36. Hansen JC (2000) Environmental contaminants and human health in the Arctic. Toxicol Lett 112-113:119-125

37. Jorgensen ME, Bjeregaard P, Borch-Johnsen K (2002) Diabetes and impaired glucose tolerance among the Inuit population of Greenland. Diabetes Care 25:1766-1771

38. Jorgensen ME, Bjerregaard P, Gyntelberg F, Borch-Johnsen K, Greenland Population Study (2004) Prevalence of the metabolic syndrome among the Inuit in Greenland. A comparison between two proposed definitions. Diabet Med 21:1237-1242

39. Yoon KH, Lee JH, Kim JW et al (2006) Epidemic obesity and type 2 diabetes in Asia. Lancet 368:1681-1688

40. Greenpeace (2000) Unseen poisons in Asia. Available from http:// www.greenpeace.to/publications pdf/asiapops.pdf, accessed 20 February 202007 